\section{Diagnostic performance of tear function tests in Sjogren's syndrome patients}

P Versura ${ }^{1}$, M Frigato ${ }^{2}, M$ Cellini' ${ }^{1}$ R Mulè ${ }^{2}$, N Malavolta ${ }^{2}$ and EC Campos ${ }^{1}$

\author{
Abstract \\ Objective To evaluate the diagnostic \\ performance of the tests included in primary \\ Sjogren's syndrome (SS-I) diagnostic criteria \\ (Schirmer I, break-up time, vital dye staining) \\ and to compare them with other examinations \\ related to the ocular surface status. \\ Methods Clinical and cytological data were \\ collected from 177 patients (62 SS-1, 56 non-SS \\ autoimmune diseases, 59 Sicca syndrome). \\ Tear tests included: a validated questionnaire \\ on symptoms, Schirmer I, Jones test, Ferning \\ test, BUT, corneal aesthesiometry, tear \\ clearance test, lissamine green staining, \\ impression conjunctival cytology. Data were \\ statistically evaluated and sensitivity, \\ specificity, likelihood ratio $(\mathrm{LR}+)$, receiver- \\ operating characteristics (ROC) curves were \\ calculated for each test. \\ Results Data showed a poor diagnostic \\ performance of Schirmer test I (sensitivity \\ 0.42; specificity $0.76 ; \mathrm{LR}+1.75$ ) and BUT \\ (sensitivity 0.92 ; specificity $0.17 ; \mathrm{LR}+1.11$ ) \\ (area under the curve in ROC analysis $<0.58$ ). \\ Validated subjective symptoms questionnaire \\ (sensitivity 0.89 ; specificity $0.72 ; \mathrm{LR}+3.18$ ), \\ Jones test (sensitivity $\mathbf{0 . 6 0}$; specificity $\mathbf{0 . 8 8}$; \\ LR +5 ), corneal aesthesiometry (sensitivity \\ 0.80 ; specificity $0.67 ; \mathrm{LR}+2.42)$, and tear \\ clearance test (sensitivity 0.63 ; specificity 0.84 ; \\ LR + 3.93), all exhibited a high diagnostic \\ performance (area under the curve in the ROC \\ analysis always $>0.70$ ). Lissamine green \\ staining exhibited the best performance \\ (sensitivity 0.63 ; specificity 0.89 ; LR +5.72 ) but \\ the result could be distorted by an \\ incorporation bias. \\ Conclusions Our data suggest to implement \\ the items for ocular signs and symptoms \\ contained in many SS-I diagnostic criteria \\ with the use of a validated questionnaire, \\ performance of Jones test, corneal
}

aesthesiometry measurement, and tear clearance rate evaluation.

Eye (2007) 21, 229-237. doi:10.1038/sj.eye.6702204; published online 6 January 2006

Keywords: Sjogren's syndrome; Schirmer tests; lissamine green dye; tear clearance; aesthesiometry; reliability

\section{Introduction}

Sjogren's syndrome (SS) is a systemic autoimmune disease that affects exocrine glands, mainly salivary and lachrymal ones, with a marked mononuclear cell infiltrate leading to progressive impairment of gland function. ${ }^{1}$ The prevalence in the population as a whole is about $0.3-1.5 \%$, but the peak incidence $(3 \%)$ occurs in the fourth and fifth decades with a female to male ratio of $9: 1 .^{2}$

Histopathology of minor salivary gland is now regarded as a 'gold standard' for the diagnosis of SS, but it is a painful procedure with small but significant proportion of unreliable results. ${ }^{3}$ Proper evaluation of SS requires systematic multidisciplinary approach for evaluation of the oral, ocular, and systemic components of the disease. There are several published criteria for the diagnosis of SS but none has still reached a general consensus. The American-European Community criteria ${ }^{4}$ appear to be the one of the most practical because it takes into consideration the multisystem nature of the disease; the criteria require that patients meet four out of six proposed items for the diagnosis of SS-I.

Subjective symptoms and tests for eye dryness are included as two distinctive items in the majority of the diagnostic sets, but little agreement on the cutoff values is present. The ocular surface is now considered as an integrated unit, ${ }^{5}$ and any dysfunction results in
${ }^{1}$ Department of Surgical Science and Transplants, Sect. Ophthalmology, Alma Mater Studiorum Università di Bologna, Bologna, Italy

${ }^{2}$ Department of Internal Medicine and Aging, Sect. Rheumatology, Alma Mater Studiorum Università di Bologna, Italy

Correspondence: P Versura, Dipartimento 'A.Valsalva' Sezione Oftalmologia, Alma Mater Studiorum Università di Bologna, Policlinico S.Orsola - Via Massarenti, 9, 40138 Bologna, Italy Tel: + 39 (51) 636 4646; Fax: + 39 (51) 342516.

E-mail: versura@ alma.unibo.it

Received: 4 March 2005 Accepted in revised form: 20 October 2005 Published online: 6 January 2006 


\begin{tabular}{lcccc}
\hline & & \multicolumn{2}{c}{ Total no. of patients $(\mathrm{n}=177)$} \\
\cline { 2 - 5 } & Male $(\mathrm{n}=16)$ & Age (years) & Female $(\mathrm{n}=161)$ & Age $($ years $)$ \\
\hline Sjogren's Syndrome $(n=62)$ & 5 & $56.1 \pm 10.2$ & 57 & $61.6 \pm 10.8$ \\
Non-Sjogren's autoimmune disease $(n=56)$ & 6 & $53.7 \pm 9.5$ & 50 & $54.8 \pm 10.8$ \\
Rheumatoid arthritis $(n=25)$ & 3 & $54.4 \pm 3.7$ & 22 & $54.9 \pm 10.9$ \\
LES $(n=11)$ & - & 52 & 11 & $56.6 \pm 8.9$ \\
Connective disorders $(n=13)$ & 1 & $53.3 \pm 5.3$ & 5 & $57.8 \pm 11.9$ \\
Sclerodermia $(n=7)$ & 2 & $55.4 \pm 2.8$ & 54 & $52.8 \pm 11.1$ \\
Sicca Syndrome $(n=59)$ & 5 & & $61.7 \pm 10.5$ \\
\hline
\end{tabular}

a scarce or unstable preocular tear film and in the presence of unrefreshed tears in which soluble mediators store up. A range of criteria have been proposed for the assessment of patients with dry eye; the most frequently used tests are the Schirmer test I and the van Bijsterveld score; some criteria are used in association with the tear film break-up time (BUT). ${ }^{6}$

Despite the fact that increasing scientific evidence suggest to include also other tests in the assessment of dry eye, ${ }^{7}$ still in the practice it is set based primarily upon a low Schirmer test I and/or BUT. The purpose of the present work was to determine the diagnostic performance of tear function tests in the differential diagnosis of SS vs other non-Sjogren's autoimmune diseases or Sicca Syndrome.

\section{Patients}

The study included 177 patients, examined during the period June 2002-December 2004 and grouped as follows:

- (SS-I) (62 subjects), diagnosed according to the American-European Consensus Group;

- Non-SS autoimmune diseases (non-SS AD) patients (56 subjects), patients with other autoimmune diseases (such as rheumatoid arthritis, systemic lupus erythematosus, sclerodermia, connective tissue disorders) but who did not satisfy the classification criteria for secondary Sjogren's Syndrome;

- Sicca syndrome (Sicca) patients (59 subjects) reporting subjective symptoms of xeroftalmia and xerostomia, without serological positivity for any autoimmune disease, and non contact lens wearers.

Demographic data of patients are reported in Table 1.

\section{Methods}

Patients were asked to answer a validated questionnaire (ocular surface disease index, OSDI) including 12 questions, related to their subjective symptoms felt the week before. The score of the questionnaire ranges from 0 to 12 (no disability), to 13-22 (light dry eye), to $23-32$ (moderate dry eye), to 33-100 (severe dry eye). ${ }^{8}$

The Schirmer tests were performed as described elsewhere ${ }^{7}$ by using sterile Schirmer strips (ContaCare Ophthalmic) without anaesthesia (Schirmer test I) or after application of oxybuprocain $0.5 \%$ (Jones test), in a room controlled for lighting (dim light room), temperature (20$22^{\circ} \mathrm{C}$ ), and humidity (40-60\%). Abnormal value was regarded as $\leq 5 \mathrm{~mm} /$ wetting after $5 \mathrm{~min}^{7}$ for Schirmer I, and $<3 \mathrm{~mm} /$ wetting after $1 \mathrm{~min}$ for Jones test. ${ }^{9}$

Tear BUT was performed as described elsewhere ${ }^{7}$ and the time of rupture $<10 \mathrm{~s}$ was considered as abnormal. Tears were collected at the outer cantus using a $5 \mu \mathrm{l}$ sterile micropipette; Ferning test was performed and scored as already described, ${ }^{10}$ score $>$ II / III was regarded as pathological.

Lissamine green staining was performed as already reported ${ }^{11}$ and scored. ${ }^{7}$ Pathological vital staining was scored as $>9 / 18$ in six conjunctival areas measured; staining was performed only in those patients without corneal epithelial defects.

Corneal sensitivity was tested with a Cochet-Bonnet aesthesiometer (Luneau, Chartres, France), making the media of values taken in five corneal points; the length of the nylon filament was converted into $\mathrm{g} / \mathrm{mm}^{2}$ pressures according to the table provided by the manufacturer; abnormal values were regarded as pressures higher than $0.57 \mathrm{~g} / \mathrm{mm}^{2} .^{12}$

Tear clearance rate was performed as already described, ${ }^{13}$ graded onto the colour of the fluorescein dye fading compared to a control staining scale dilution; abnormal values were regarded as a staining less than or equal to $1 / 8$ dilution.

Conjunctival impression cytology was performed, processed, and scored as reported; ${ }^{14}$ the abnormal grade was regarded as $>1$.

\section{Statistical analysis}

Data were statistically evaluated by applying the Statistical Package for the Social Sciences (SPSS) version 
9.0 for the independent sample $t$-test, the Mann-Whitney test for unpaired data, and the logistic regression for selected groups of tests. For nonparametric data (Ferning test, imprint cytology, clearance test, Vital Dye Staining), the descriptive statistics applied were the analysis of median and 25-75 percentiles. Significant results values for $P$ less than 0.05 were regarded as statistically significant.

The prevalence of the SS-I (the proportion of patients who have the disease in the population under testing) was calculated having, as a reference, the population included in our study. Each of the test performed were analysed for sensitivity (the percentage of symptomatic patients who tested positive, a large sensitivity means that a negative test can rule out the disease) and specificity (the percentage of normal subjects who tested negative, a large specificity means that a positive test can rule in the disease). Specificity and sensibility were calculated comparing SS-I patients vs Sicca-patients. Data were also elaborated by using the MedCalc version 5.0 in order to calculate receiver-operating characteristics (ROC) curves, ${ }^{15}$ ROC curves express the diagnostic accuracy of a test variable by plotting the sensitivity of the test against the specificity at all possible thresholds.

We also summarized the data about diagnostic tests using the likelihood ratio, a measure that combines information about the sensitivity and specificity, and provides a direct estimate of how much a positive or negative result changes the likelihood that a patient would have the disease. The likelihood ratio for a positive result (LR + ; sensitivity divided by 1-specificity) indicates how much the odds of the disease increase when a test is positive.

\section{Results}

Table 2 summarizes the medium $\pm S D$ of the values resulted from the study, collected from each group of patients. Data are also shown in separate graphs and discussed hereby.
Dry eye symptoms were reported by all patients (score of the subjective symptom questionnaire always $>12$ ), ranging from moderate in Sicca to severe in SS-I and nonSS AD patients, with statistically significant differences (Graph 1) between SS-I and the other two groups of patients.

Medium values of pathological Schirmer test I (paper wetting $<5 \mathrm{~mm} / 5 \mathrm{~min}$ ) were never found in any group (Table 2), even in the SS-I patients and with no statistically significant differences among the groups (Graph 2).

Medium values of Jones test showed a pathological decrease of tear production only in the SS-I patients (Table 2), with statistically significant differences with the other two groups (Graph 3).

Both Ferning test (Table 3) and BUT (Graph 4) showed pathological values in all the three groups (Table 2),

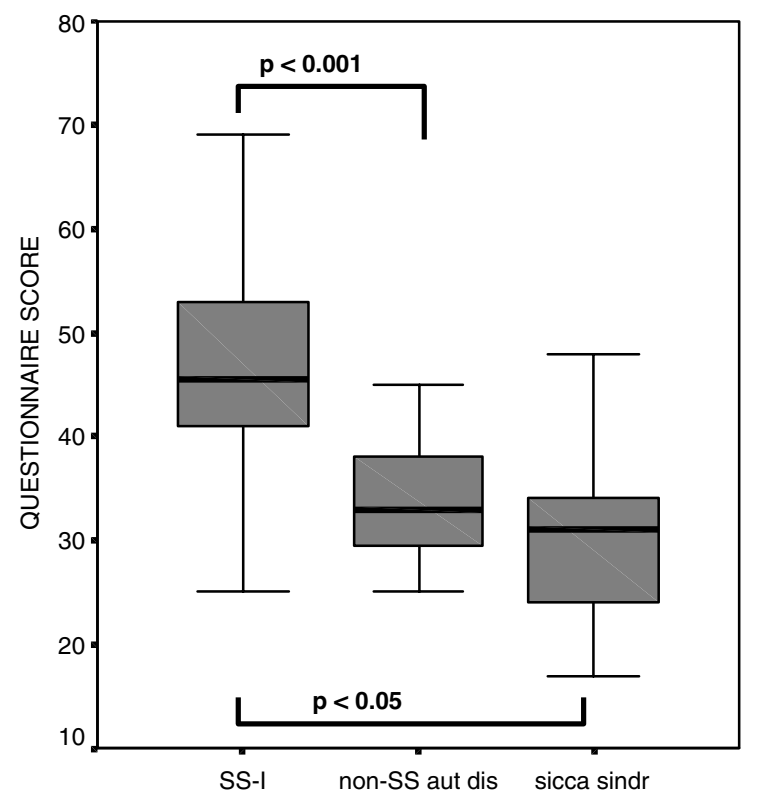

Graph 1 Questionnaire score.

Table 2 Summary of the results (medium \pm SD)

\begin{tabular}{llccc}
\hline Test & Measure & SS-I & Non-SS aut dis & Sicca Sindr \\
\hline Questionnaire & Score & $47 \pm 10$ & $34 \pm 5$ & $30 \pm 7$ \\
Schirmer test I & $\mathrm{mm} / 5^{\prime}$ & $8.8 \pm 7.4$ & $10.5 \pm 6.7$ & $11.1 \pm 7.3$ \\
Jones test & $\mathrm{mm} / 1^{\prime}$ & $2.9 \pm 2.3$ & $5.4 \pm 3$ & $6.5 \pm 3$ \\
Ferning test* & Grade I/IV & $2.8 \pm 0.8$ & $2.7 \pm 0.7$ & $2.9 \pm 0.6$ \\
BUT & Seconds & $4.3 \pm 3.5$ & $5.1 \pm 4.3$ & $5.7 \pm 3.9$ \\
Clearance test** & Score & $3.2 \pm 1.2$ & $2.5 \pm 0.9$ & $1.9 \pm 0.9$ \\
Corneal aesthesiometry & g/mm & $0.88 \pm 0.12$ & $0.56 \pm 0.1$ & $0.52 \pm 0.08$ \\
Vital staining (lissamine green) & Score & $10 \pm 3.1$ & $7.4 \pm 4.2$ & $6.7 \pm 4.2$ \\
Imprint cytology & Score & $1.9 \pm 0.8$ & $1.6 \pm 0.7$ & $1.2 \pm 0.5$ \\
\hline
\end{tabular}

For the statistical analysis the following results were converted into numerical values: * grade I = 1; grade I/II =1,5; grade II =2; grade II/III =2,5; grade $\mathrm{III}=3$; grade III $/ \mathrm{IV}=3,5$; grade IV $=4 .{ }^{* *}$ diluition $1: 2=5 ; 1: 4=4 ; 1: 8=3 ; 1: 16=2 ; 1: 32=1 ; 1: 64=0$. 


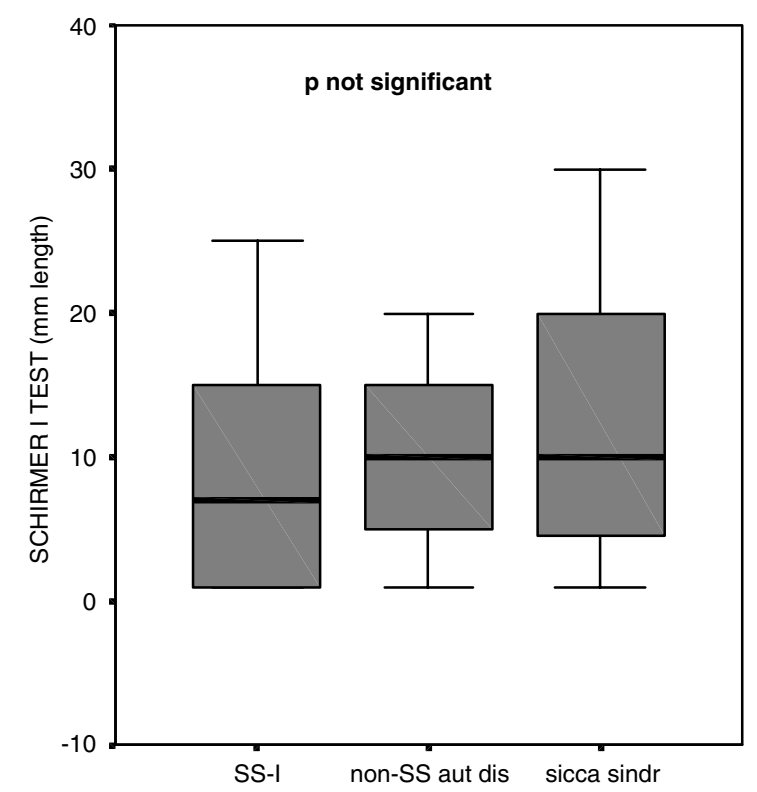

Graph 2 Schirmer test.

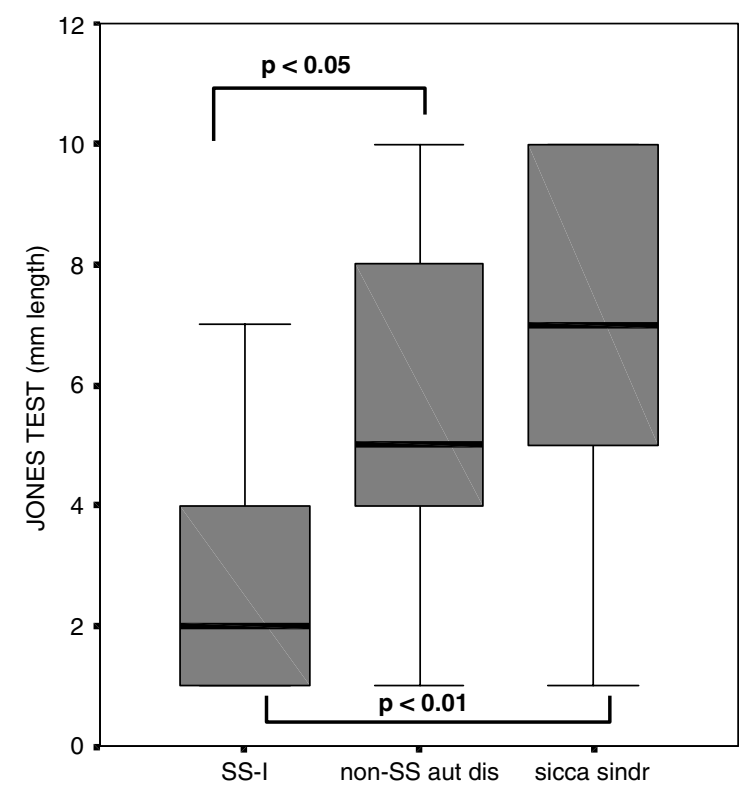

Graph 3 Jones test.

without statistically significant differences among the groups.

Medium values of clearance test and corneal aesthesiometry (Table 2) showed, respectively, a pathological reduction of tear turnover (Table 4) and corneal sensitivity (Graph 5) only in the SS-I patients (Table 2), with statistically significant differences with the other two groups.

The vital dye staining score resulted in the pathological range only in the SS-I patients (Table 2), with statistically
Table 3 Analysis of median and percentiles for ferning test

\begin{tabular}{lccc}
\hline & Median & Percentiles 25 & Percentiles 75 \\
\hline SS-I & 3.0 & 2.0 & 3.5 \\
Non-SS aut dis & 3.0 & 2.5 & 3.0 \\
Sicca Sindr & 3.0 & 2.5 & 3.0 \\
\hline
\end{tabular}

$P$ not significant

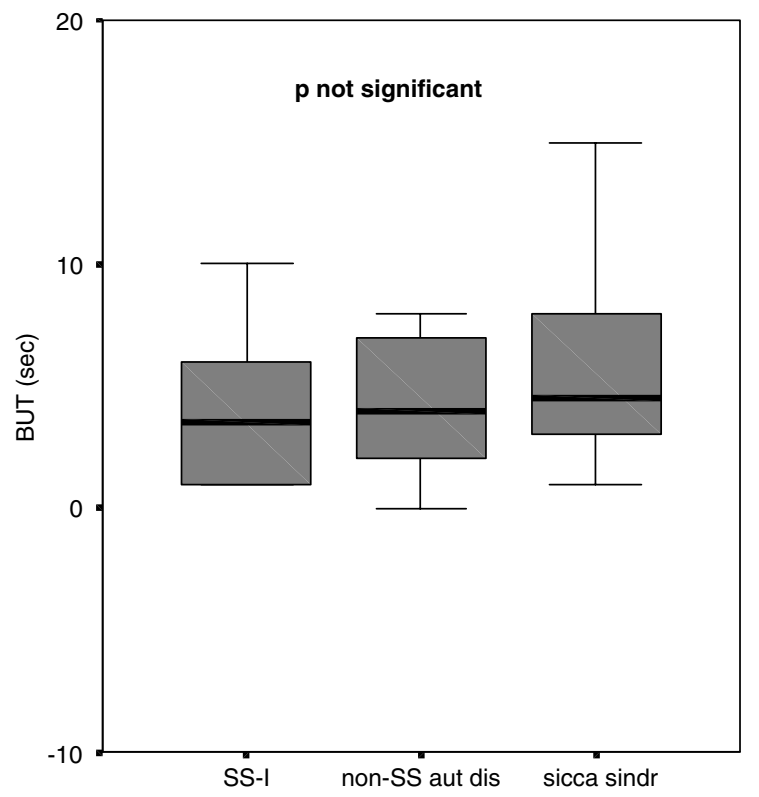

Graph 4 Break-up time.

Table 4 Analysis of median and percentiles for clearance test

\begin{tabular}{|c|c|c|c|}
\hline & Median & Percentiles 25 & Percentiles 75 \\
\hline$*[[$ SS-I & 4.0 & 2.75 & 4.0 \\
\hline Non-SS aut dis & 2.5 & 2.0 & 3.0 \\
\hline$*$ Sicca Sindr & 2.0 & 1.0 & 3.0 \\
\hline
\end{tabular}

significant differences with the other two groups (Table 5).

The conjunctival imprint cytology score demonstrated pathological values in all the three groups, more severe in the SS-I patients, and with statistically significant differences between SS-I and the other two groups of patients (Table 6).

Table 7 summarizes the sensitivity, specificity, and positive likelihood ratio, for each test. Diagnostic performance of the tests will be shown and discussed further. 
In our study, the Schirmer test I performed poorly as a diagnostic test for SS-I (sensitivity 0.42 ; specificity 0.76 , LR + 1.75) and ROC plot analyses (Figure 1a) demonstrated a flat curve, running parallel to the diagonal line (are under the curve 0.412), which also indicates low accuracy of the test.

The BUT also performed poorly as a diagnostic test for SS (sensitivity 0.92 but specificity only 0.17 ; LR +1.11 );

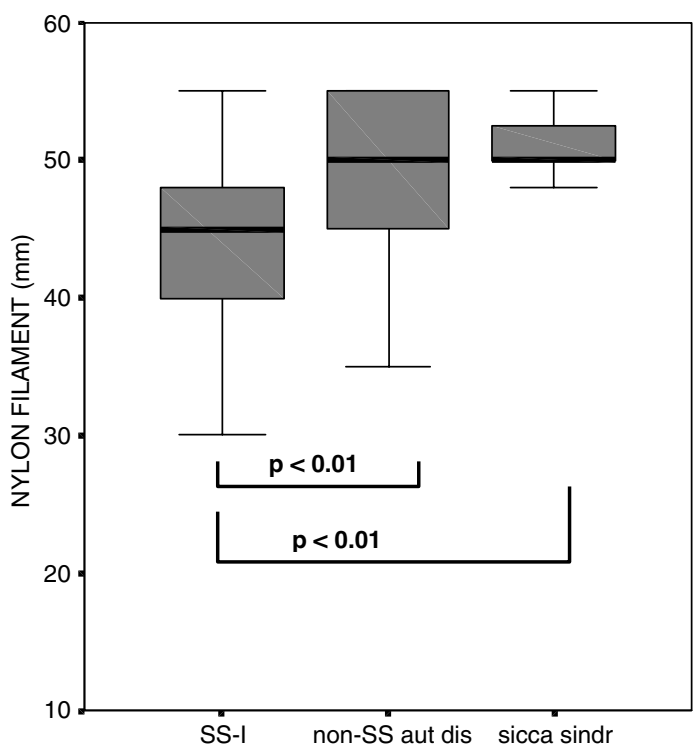

Graph 5 Corneal aesthesiometry.

Table 5 Analysis of median and percentiles for lissamine green staining

\begin{tabular}{|c|c|c|c|}
\hline & Median & Percentiles 25 & Percentiles 75 \\
\hline$*[[$ SS-I & 10.0 & 10.0 & 10.0 \\
\hline L Non-SS aut dis & 10.0 & 3.35 & 10.0 \\
\hline$*$ L Sicca Sindr & 10.0 & 2.75 & 10.75 \\
\hline
\end{tabular}

${ }^{*} P<0.01$.
ROC plot analyses (Figure 1b) also indicated low accuracy of the test (area under the curve 0.597 and flat curve).

The vital dye staining performed better than the other two previous tests (sensitivity 0.63 ; specificity 0.89 ; LR + 5.72); ROC plot analyses demonstrated a curve slightly approaching the upper left corner of the diagram (Figure 1c) and a larger area under the curve (0.680), which indicates an increasing diagnostic performance of the test.

In the present study, the tests that showed the best performance were the validated subjective symptom questionnaire (sensitivity 0.89 ; specificity $0.72 ; \mathrm{LR}+3.18$; area under the curve in the ROC plot analyses 0.896 ; Figure $2 \mathrm{a}$ ), the Jones test (sensitivity 0.60 ; specificity 0.88 ; $\mathrm{LR}+5$; area under the curve in the ROC plot analyses 0.703 ; Figure $2 b$ ), the corneal aesthesiometry (sensitivity 0.80 ; specificity $0.67 ; \mathrm{LR}+2.42$; area under the curve in the ROC plot analyses 0.674; Figure 2c), and the tear clearance test (sensitivity 0.63 ; specificity 0.84 ; LR +3.93 ; area under the curve in the ROC plot analyses 0.754 ; Figure 2d). In Figure 2(a-d), all the ROC-curve analyses of these tests are collected: all the curves show the tendency to approach the upper left corner of the diagram, especially the curve related to questionnaire data (Figure 2a) and tear clearance test (Figure 2d), indicating high diagnostic performance.

Further support to our data comes from the logistic regression analysis between SS-I and Sicca Syndrome

Table 6 Analysis of median and percentiles for imprint cytology

\begin{tabular}{lccc}
\hline & Median & Percentiles 25 & Percentiles 75 \\
\hline * & 2.0 & 1.0 & 2.5 \\
SS-I & 1.5 & 1.0 & 2.0 \\
* Non-SS aut dis & 1.0 & 1.0 & 1.75 \\
\hline
\end{tabular}

$* P<0.05$.

Table 7 Sensibility, specificity, likelihood ratio for each test, calculated comparing SS-I vs Sicca Syndrome patients, listed on increasing value of $\mathrm{LR}+$

\begin{tabular}{|c|c|c|c|c|}
\hline Test & $\begin{array}{l}\text { Pathological } \\
\text { value }\end{array}$ & Sensibility & Specificity & Likelihood ratio $(L R+)$ \\
\hline Ferning test & $>$ Grade II/III & 0.75 & 0.10 & 0.83 \\
\hline Imprint cytology & Score $>1$ & 0.94 & 0.10 & 1.04 \\
\hline BUT & $<10 \mathrm{~s}$ & 0.92 & 0.17 & 1.11 \\
\hline Schirmer test I & $\leq 5 \mathrm{~mm} / 5^{\prime}$ & 0.42 & 0.76 & 1.75 \\
\hline Corneal esthesiometry & $>0.57 \mathrm{~g} / \mathrm{mm}^{3}$ & 0.80 & 0.67 & 2.42 \\
\hline Questionnaire & Score $>33$ & 0.89 & 0.72 & 3.18 \\
\hline Clearance test & Diluition $\geq 3$ & 0.63 & 0.84 & 3.93 \\
\hline Jones test & $<3 \mathrm{~mm} / 1^{\prime}$ & 0.60 & 0.88 & 5 \\
\hline Vital staining & Score $>8 / 18$ & 0.63 & 0.89 & 5.72 \\
\hline
\end{tabular}

Explanation in the text, sections Methods and Discussion. 


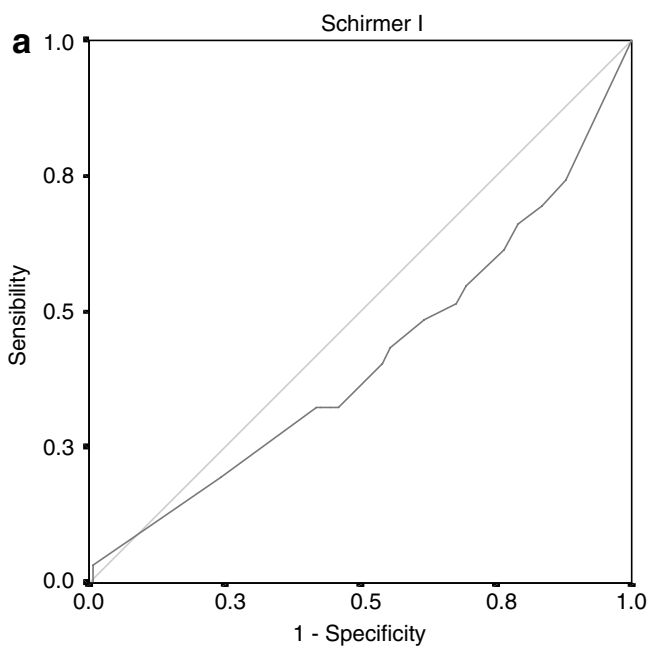

Area under the curve $=0.412$ Standard error $=0.046$ $95 \% \mathrm{Cl}=0.322-0.503$

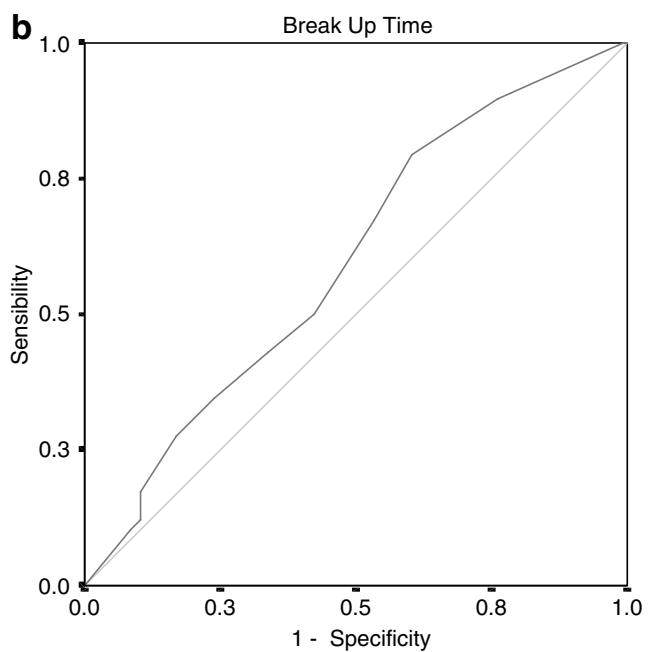

Area under the curve $=0.597$ Standard error $=0.044$ $95 \% \mathrm{Cl}=0.510-0.684$

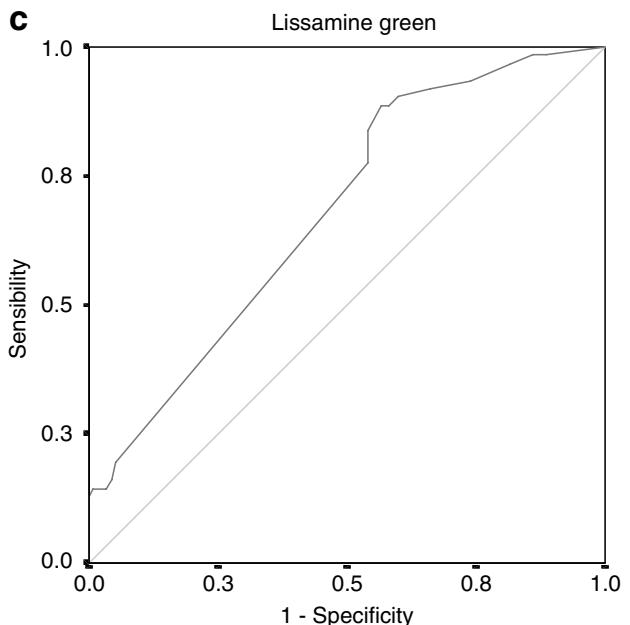

Area under the curve $=0.680 \quad$ Standard error $=0.041$ $95 \% \mathrm{Cl}=0.600-0.759$
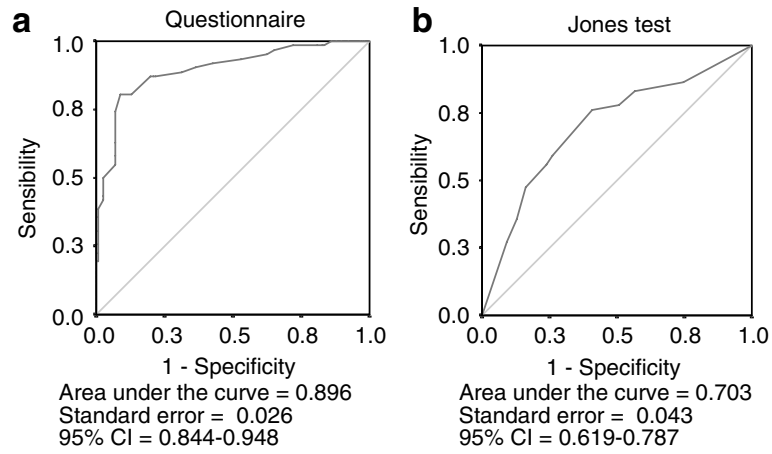

Area under the curve $=0.703$ Standard error $=0.043$ $95 \% \mathrm{Cl}=0.619-0.787$

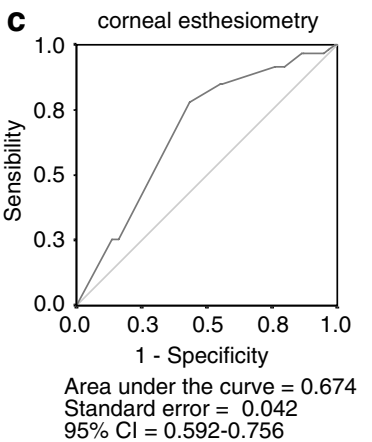

d tear clearance test

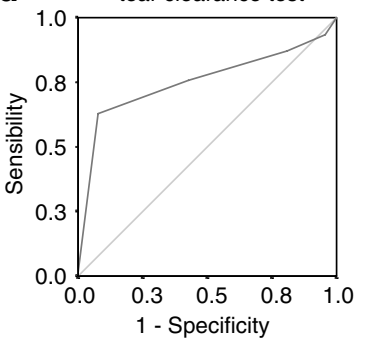

Area under the curve $=0.75$ Standard error $=0.044$ $95 \% \mathrm{Cl}=0.667-0.840$

Figure 2 Nonparametric ROC-curves of tear tests evaluated for their use in identifying SS-I patients. Note the relative relative rounded curve (towards the upper left corner of the diagram) of all the four tests, indicating good diagnostic performance.

patients performed for Jones test, corneal aesthesiometry, symptom questionnaire, and clearance test as a whole (respectively, $P=0.003, P=0.015, P=0.0001, P=0.034$ ), demonstrating the key role of these tests in the differential diagnosis.

\section{Discussion}

Dry eye often affects patients suffering from a variety of rheumatic diseases, especially SS-I. The ocular surface status has been included in almost all the diagnostic criteria for SS-I, in items separate for subjective symptoms and clinical assessment, including the Schirmer test I, the BUT and a surface staining with vital dyes (Bengal Rose or Lissamine green).

Despite the relevance of the appropriate use of diagnostic tests in clinical decisions, many tests have not yet been subjected to evaluation to establish their clinical utility. There is much debate about the usefulness and accuracy of the Schirmer test I; some authors

Figure 1 Nonparametric ROC-curves of tear tests evaluated for their use in identifying SS-I in patients. Note the relative flat curves of all the three tests, indicating poor diagnostic performance. 
demonstrated that it is a reliable test for the diagnosis of SS- $\mathrm{I}^{16}$ while others discussed its role ${ }^{17}$ showing that Schirmer test I has a moderate repeatability from visit to visit ${ }^{18}$ and displays only a weak association with subjective symptoms of dryness. ${ }^{19}$ It can probably be assumed that the Schirmer test is of little value for mild to moderate dry eyes and only a very low score of a Schirmer test I can be regarded as a good indication that there is in fact an aqueous deficiency. The cutoff for Schirmer I is wetting $\leq 5 \mathrm{~mm} / 5 \mathrm{~min}$ in the AmericanEuropean criteria for SS-I, but in the present study, the medium value for Schirmer test I was not found under this cutoff in the SS-I group and no statistically significant difference among the groups was found, in agreement with others. ${ }^{20}$ The item for ocular signs in these criteria is satisfied when there is objective evidence of eye involvement as a positive result for at least one out of two tests between Schirmer I and Rose Bengal staining; SS-I diagnosis can then be reached when patients meet four out of six item. ${ }^{4}$ It is conceivable that a significant part of SS-I patients included in the present study had met the ocular sign item with the positivity of the Rose Bengal staining only, or that at the time of our visit, Schirmer results were influenced by the stage of the disease and/or by the therapy. ${ }^{1}$

Our data showed that diagnostic performance of the Schirmer test I is poor, but no definitive conclusion can be drawn from this study, since the same cutoff had been used to support the diagnoses by which patients were grouped and, therefore, the calculated sensitivity and specificity for Schirmer test are flattered by an incorporation bias. It can be concluded, however, that the Schirmer criterion seem to produce many false negative test results (about half of the SS-I patients tested in our study were negative for Schirmer I) in agreement with others. ${ }^{17}$

Data from the present study demonstrated that a negative BUT can exclude diagnosis of SS-I (high sensitivity 0.97 ) but with low specificity: in all the three groups of patients, the BUT medium value was in the pathological range with no statistically significant differences among the groups in agreement with others; ${ }^{20}$ the scarce diagnostic performance of BUT in SS-I diagnosis was also showed by the flat curve slightly above the diagonal line in the ROC plot analysis.

The vital dye (Lissamine green) staining showed statistically significant differences between the SS-I patients and the other groups, and a high specificity, indicating that a positive result can rule in the SS-I diagnosis. Although the vital staining exhibited the highest likelihood ratio in this study, in agreement with previous works that indicated vital dye score the test of choice, ${ }^{21}$ the complessive diagnostic performance was scarce in our study, as it can be seen in the ROC plot analysis flat curve, probably as a consequence of a bias in data collection, since vital dye staining was applied only to those patients with Fluo negative corneas.

The imprint cytology is a noninvasive alternative to the full-thickness biopsy for the collection of epithelial cells from the ocular surface, with no side effects or contraindications. ${ }^{22}$ The grading system is based upon cytological parameters ${ }^{5}$ and the clinical severity of the dry eye correlates with these alterations. ${ }^{23}$ Imprint cytology showed the highest sensibility among the diagnostic tests performed in the population under study but also the lower specificity and a low LR + . This means that the cytology indicating ocular surface dryness is a common finding in all the three groups of our patients, a result that makes imprint cytology a poor candidate to perform satisfactorily as a tool in SS-I differential diagnosis.

Data from the present study demonstrated that measures performing better in the SS-I diagnosis are the OSDI validated questionnaire, the Schirmer test with anaesthesia, the corneal aesthesiometry, and the tear clearance test, all showing good LR + values and curve shapes in the ROC plot analysis. Taken together and analysed with the logistic regression method, they were demonstrated to significantly contribute to the prediction of the SS-I diagnosis.

The item for ocular subjective symptoms only includes few simple questions concerning the common feeling of sandy eyes, eye discomfort, and use of tear substitutes, in the majority of the SS-I diagnostic criteria, including the American-European we used for the study. The use of a validated Sicca symptoms questionnaire was already demonstrated a useful tool, ${ }^{24}$ and the score of ocular discomfort based on multiple questions, a measure that more precisely distinguish SS-I patients from non-SS AD patients. ${ }^{25}$ The OSDI validated questionnaire ${ }^{8}$ adopted in our lab routine and in the present study demonstrated high specificity and exhibited the best diagnostic performance, indicating that a scored symptom investigation plays a primary role in orienting the clinician to make a SS-I diagnosis.

The Schirmer test I is used widely to measure total tear secretion, traditionally subdivided into a basal vs a reflex component, although this classification lost much of its importance over the last years as it will be discussed further. Among the modified forms of Schirmer tests, the Schirmer test with nasal stimulation appears a reliable measure of maximal tear secretion, able to recognize ocular surface impairment in many diseases ${ }^{26}$ and it is recommended to screen potential SS-I patients before expensive serological tests. The test is performed using longer Schirmer paper strips with or without the use of 
topical anaesthesia, with a cotton swab kept in place into the patient's nasal cavity at the second turbinate level; the test reflects the lachrymal gland condition and relates to lymphocytic infiltration of the lachrymal gland and the severity of the disease. ${ }^{27}$ In our experience, the nasal stimulation was applied, but too often scarcely tolerated by subjects, and in our clinical practice, we preferred to choose the Schirmer test with topical anaesthesia (Jones test) to exclude tear secretion coming from external stimuli, the so-called basal tears. Jones test showed in our study a high specificity and a good performance in the SS-I differential diagnosis, with statistically significant different values between the SS-I and the other two groups of patients, suggesting to perform it in association with Schirmer test I.

Actually, extensive scientific evidence now recognizes that all tears are under tight neural control in order to respond rapidly to changing inner and/or outer conditions. The updated concepts on tear physiology ${ }^{28,29}$ state that tear secretion is controlled by a neural reflex arc that integrates the function of all the components of the ocular surface unit: ${ }^{5}$ a neural arc uses ophthalmic branch of the trigeminal nerve, driven by corneal sensitivity, as afferent way and the facial nerve as efferent way; the final neuroanatomic integration of these two reflexes is manifested in tear clearance. ${ }^{30}$

The measurements of corneal sensitivity, measured by aesthesiometry, and tear clearance rate are now currently considered as integrated measurements to analyse the ocular surface as a whole. Results from the present study demonstrated a reduction of both these parameters in the SS-I patients with statistically significant differences vs the two other groups. In the SS-I patients, the chronic presence of inflammation caused by the lymphocytic infiltration of the lachrymal gland reduces tear secretion and corneal sensitivity, ${ }^{31}$ as also found by others ${ }^{32}$ and supported by morphological findings. ${ }^{33}$ A reduced tear secretion, consequent to reduced corneal sensitivity, could also be in relation with an impairment in the parasympathetic control of lachrymal gland, as demonstrated in other exocrine gland, ${ }^{34,35}$ as a consequence of the presence of anti M3 muscarinic receptors ${ }^{36}$ independently from the concurrent presence of anti-SSA and anti-SSB, ${ }^{37}$ as it has been demonstrated in an animal model..$^{38}$

A reduced tear clearance rate results in accumulation of corneal metabolism products, including the inflammatory cytokines present in the tear fluid of SS-I patients $^{39,40}$ maintaining the ocular surface inflammation within a vicious circle.

In conclusion, and as a final remark, a diagnostic test gives the clinician an indication of whether a patient has a certain disease; the lack of good quality research in the field contributes to inappropriate utilization and significant wasting of resources. Data from the present study suggest to implement the items of subjective symptoms and objective evaluations contained in the majority of SS-I diagnostic criteria. In particular, our results confirm the scarce reliability of Schirmer test I and BUT and discuss the role of vital dye staining as the test of choice in the SS-I differential diagnosis. It is suggested to implement the diagnostic route with the use of a validated questionnaire, the performance of Schirmer test with anaesthesia, the measurement of corneal aesthesiometry, and the analysis of tear clearance rate, all tests that showed the highest diagnostic performance in the present study and population.

\section{Acknowledgements}

We are indebted to Mrs Chiara Coslovi, for her technical assistance and kindness with all of us.

\section{References}

1 O'Day DM, Horn JD. The eye and rheumatic disease. In: Kelley WN (ed). Textbook of Rheumatology, 6th ed. Saunders: Philadelphia, 2001 Chapter 29.

2 Thomas E, Hay EM, Hajeer A, Silman AJ. Sjogren's syndrome: a community-based study of prevalence and impact. Br J Rheum 1998; 37: 1069-1076.

3 Martin-Martin LS, Latini A, Pagano A, Ragno A, Stasi R. A new mathematical model based on clinical and laboratory variables for the diagnosis of Sjogren's syndrome. Clin Rheumatol 2003; 22: 123-126.

4 Vitali C, Bombardieri S, Jonsson R, Moutsopoulos HM. Classification criteria for Sjogren's syndrome: a revised version of the European criteria proposed by the American-European Consensus Group. Ann Rheum Dis 2002; 61: 554-558.

5 Tseng SC, Tsubota K. Important concepts for treating ocular surface and tear disorders. Am J Ophthalmol 1997; 124: 825-835.

6 Prause JU. Clinical ophthalmological tests for the diagnosis of keratoconjunctivitis sicca. Clin Exp Rheumatol 1989; 7: 141-144.

7 Lemp MA. Report of the national eye institute/industry workshop on clinical trials in dry eyes. CLAO J 1995; 21: 221-232.

8 Schiffman RM, Christanson MD, Jacobsen G. Reliability and validity of the ocular surface disease index. Arch Ophthalmol 2000; 118: 615-621.

9 Bawazeer AM, Hodge WG. One-minute Schirmer test with anaesthesia. Cornea 2003; 22: 285-287.

10 Ravazzoni L, Ghini C, Macri A, Rolando M. Forecasting of hydrophilic contact lens tolerance by means of tear ferning test. Graefe's Arch Clin Exp Ophthalmol 1998; 236: 354-358.

11 Chodosh J, Dix R, Howell RC, Dtroop WG, Tseng SC. Staining characteristics and antiviral activity of sulphorodamine B and lissamine green B. Invest Ophthalmol Vis Sci 1994; 35: 1046-1058.

12 Rosenberg ME, Tervo TM, Immonen IJ, Muller IJ, Vesaluoma MH. Corneal structure and sensitivity in type 1 
diabetes mellitus. Invest Ophthalmol Vis Sci 2000; 41: 2915-2921.

13 Xu KE, Yagi Y, Toda I, Tsubota K. Tear function index. A new measure of dry eye. Arch Ophthalmol 1995; 113: 84-88.

14 Tseng SCG. Staging of conjunctival squamous metaplasia by impression cytology. Ophthalmology 1985; 92: 728-733.

15 Zweig MH, Campbell R. Receiver-Operating Characteristic (ROC) Plot. A fundamental evaluation tool in clinical medicine. Clin Chem 1993; 39: 561-577.

16 Vitali C, Moutsopoulos HM, Bombardieri S. The European Community Study Group on diagnostic criteria for Sjogren's syndrome. Sensitivity and specificity of tests for ocular and oral involvement in Sjogren's syndrome. Ann Rheum Dis 1994; 53: 637-647.

17 Haga HJ, Hulten B, Bolstad AI, Ulvestad E, Jonsson R. Reliability and sensitivity of diagnostic tests for primary Sjogren's syndrome. J Rheumatol 1999; 26: 604-608.

18 Nichols KK, Mitchell GL, Zadnik K. The repeatability of clinical measurements of dry eye. Cornea 2004; 23: 272-285.

19 Hay EM, Thomas E, Pal B. Weak association between subjective symptoms or and objective testing for dry eyes and dry mouth: results from a population based study. Ann Rheum Dis 1998; 57: 20-24.

20 Tsubota K, Toda I, Yagi Y, Ogawa Y, Ono M, Yoshino K. Three different types of dry eye syndrome. Cornea 1994; 13: 202-209.

21 Vissing A, Kalk WW, Mansour K, Spijkervet FK, Bootsma H. Comparison of lachrymal and salivary gland involvement in Sjogren's syndrome. Arch Otolaryngol Head Neck Surg 2003; 129: 966-971.

22 Calonge M, Diebold Y, Saez V. Impression cytology of the ocular surface: a review. Exp Eye Res 2004; 78: 457-472.

23 Rivas L, Murube J, Shalaby O, Oroza MA, Sanz AI. Impression cytology contribution to differential diagnosis of Sjogren's syndrome in the ophthalmological clinic. Arch Soc Esp Oftalmol 2002; 77: 63-72.

24 Brun JG, Jacobsen H, Kloster R, Cuida M, Johannesen AC, Hoyeraal HM et al. Use of a sicca symptoms questionnaire for the identification of patients with Sjogren's syndrome in a heterogeneous hospital population with various rheumatic diseases. Clin Exp Rheumatol 1994; 12: 649-652.

25 Bowman SJ, Booth DA, Platts RG. Validation of the Sicca Symptoms Inventory for clinical studies of Sjogren's syndrome. J Rheumatol 2003; 30: 1259-1266.

26 Tsubota K, Kaido M, Yagi Y, Fujihara T, Shimmura S. Diseases associated with ocular surface abnormalities: the importance of reflex tearing. Br J Ophthalmol 1999; 83: 89-91.
27 Tsubota K, Xu KP, Fujihara T, Katagiri S, Takeuchi T. Decreased reflex tearing is associated with lymphocytic infiltration in lachrymal glands. J Rheumatol 1996; 23: 313-320.

28 Sangwan VS, Tseng SCG. New perspectives in ocular surface disorders. An integrated approach for diagnosis and management. Indian J Ophthalmol 2001; 49: 153-168.

29 Hodges RR, Dartt DA. Regulatory pathways in lachrymal gland epithelium. Int Rev Cytol 2003; 231: 129-196.

30 Sade de Paiva C, Plugfelder SC. Tear clearance implications for ocular surface health. Exp Eye Res 2004; 78: 395-397.

$31 \mathrm{Xu} \mathrm{KP}$, Yagi Y, Tsubota K. Decrease in corneal sensitivity and change in tear function in dry eye. Cornea 1996; 15: 235-239.

32 Adatia FA, Michaeli-Cohen A, Naor J, Caffery B, Bookman A, Slomovic A. Correlation between corneal sensitivity, subjective dry eye symptoms and corneal staining in Sjogren's syndrome. Can J Ophthalmol 2004; 39: 767-771.

33 Tuominen ISJ, Konttinen YT, Vesaluoma MH, Moilanen JAO, Helinto M, Tervo TMT. Corneal innervation and morphology in primary Sjogren's Syndrome. Invest Ophthalmol Vis Sci 2003; 44: 2545-2549.

34 Kovacs L, Torok T, Bari F. Impaired microvascular response to cholinergic stimuli in primary Sjogren's syndrome. Ann Rheum Dis 2000; 59: 48-53.

35 Kovacs L, Papos M, Takacs F. Autonomic nervous system dysfunction involving the gastrointestinal and the urinary tracts in primary Sjogren's syndrome. Clin Exp Rheumatol 2003; 21: 697-703.

36 Jonsson R, Haga HJ, Gordon TP. Current concepts on diagnosis, autoantibodies and therapy in Sjogren's syndrome. Scand J Rheumatol 2000; 29: 341-348.

37 Bacman S, Berra A, Sterin-Borda L, Borda E. Muscarinic acetylcholine receptor antibodies as a new marker of dry eye Sjogren's syndrome. Invest Ophthalmol Vis Sci 2001; 42 321-327.

38 Bacman S, Sterin-borda L, Camusso JJ. Circulating antibodies against rat parotid gland M-3 muscarinic receptors in primary Sjogren's syndrome. Clin Exp Immunol 1996; 104: 454-459.

39 Rolando M. Sjogren's syndrome as seen by an ophthalmologist. Scand J Rheumatol 2001; 115(suppl): 27-33.

40 Solomon A, Dursun D, Liu Z. Pro- and anti-inflammatory forms of interleukin-1 in the tear fluid and conjunctiva of patients with dry eye disease. Invest Ophthalmol Vis Sci 2001; 42: 2283-2292. 